\title{
Wireless Nonorthogonal Chaotic Communications: Opportunities, Challenges, and Future Directions
}

\author{
Israa Almusawi ${ }^{1}$, Walid Al-Hussaibi ${ }^{2}$, and Yaseen H. Tahir ${ }^{3}$ \\ \{israa.almusawii@gmail.com ${ }^{1}$, alhussaibi@stu.edu.iq ${ }^{2}$,yaseen_tcb@stu.edu.iq $\left.{ }^{3}\right\}$ \\ $\left\{\mathrm{FGS}^{1}, \mathrm{BTI}^{2}, \mathrm{QTI}^{3}\right\}$, Southern Technical University, Basrah, Iraq
}

\begin{abstract}
The new communication networks are expected to support the growing demands for wireless services with critical requirements of high connectivity, spectral efficiency, reliability, and information security. Therefore, nonorthogonal multiple access (NOMA) strategy has been adopted as a key component to fulfill the main targets of 5G systems. On the other hand, varied chaotic communication systems have been introduced for robust and cost-effective physical layer security. This paper presents a condensed survey on the existing chaos-based communications and NOMA schemes with their important features and applications. Then, the investment of chaotic signals in NOMA is highlighted as a promising wireless nonorthogonal chaotic communication (WNCC) technology. An insightful vision on the possible opportunities of WNCC schemes based on code and/or power domains is presented and verified by the available findings and one of our preliminary paradigms to demonstrate the extended connectivity with desired security. Moreover, the major challenges and practical considerations for WNCC systems are discussed and followed by some avenues for future research directions.
\end{abstract}

Keywords: Chaotic communications; NOMA schemes; code-domain NOMA; powerdomain NOMA; wireless channel; physical layer security.

\section{Introduction}

With the explosive developments and deployments of wireless technologies, the number of connected devices like smartphones, personal computers, and home appliances is expected to be increased on a massive scale worldwide. Therefore, numerous communication system designs are investigated in the literature to fulfill the main requirements of new and future wireless networks like high user connectivity, ultra-reliability, low complexity, affordable complexity, and robust information security [1]-[7]. In particular, nonorthogonal multiple access (NOMA) is considered as a key element to realize the main targets of fifth-generation $(5 \mathrm{G})$ wireless systems and beyond. Based on the power-domain or code-domain, NOMA can outperform the conventional orthogonal multiple access (OMA) techniques in terms of user connectivity and spectral efficiency without significant loss in error performance [8]-[16]. Physical layer security concerns are also tackled in NOMA schemes by employing chaotic signals in the code-domain [7], [17]-[19], or the inherent inter-user interference [20].

In the literature, different chaos-based secure communication (CBSC) systems have been introduced by intensive research works to realize robust and cost-effective data security for varied single-user and multiuser wireless applications [21]-[28]. The efficacy of these schemes is realized by exploiting the attractive characteristics of chaos signals like the simple generation using low-priced circuits, noise-like spectrum, unpredictable behaviour, and sensitivity to initial 
conditions (ICs) [24], [25]. For such CBSC systems, the transmission of private data is usually buried into the utilized chaotic signal and emitted to the receiver over a wireless channel. Chaos synchronization between the transmitter and receiver sides is essential incoherent systems to estimate the transmitted data [28]. Nevertheless, this condition is not required for non-coherent detection where data estimation can be carried out through the observed characteristics of the received signal [21], [29].

In the last years, CBSCs have been integrated with NOMA approaches to achieve most of the well-known gains of these interesting technologies [7]-[19], [30]. Thus, our focus in this paper is to provide a condensed survey on the existing contributions in the areas of CBSCs and NOMA with their features, results, and main applications. Then, the investment of simplegenerated chaos signals in NOMA schemes is investigated as a promising wireless nonorthogonal chaotic communication (WNCC) strategy with the possible opportunities and future directions. The main contributions of this work are highlighted as follows:

1) A relevant and condensed technical background on the existing CBSCs and NOMA methods is introduced for the interested readers from different research areas and to pave the way for a better understanding of the integrated WNCC systems.

2) An insightful vision on the possible opportunities of WNCC schemes based on code and/or power domains is presented with the available findings. An example from our preliminary results of code-domain WNCC paradigm is given also to demonstrate the extended multiuser connectivity with desired information security.

3) The major challenges and practical issues for promising WNCCs are discussed and followed by potential directions to extend the state-of-the-art for future applications.

The rest of this paper is organized as follows. Section 2 provides a technical background review on the investment of chaos in communication systems while a review of the NOMA schemes is presented in Section 3. The main principles and our perspective for WNCC systems are demonstrated in Sections 4. Research challenges and practical considerations for WNCC are highlighted in Section 5 while some of the future research directions are given in Section 6. Finally, the paper is concluded in Section 7.

\section{Chaos in Communication Systems}

Chaos has taken great attention in the past few decades and has been invested in a variety of applications like signal processing, cryptography, secure communications, and multiple access communications [21], [28]-[34]. The important topics of CBSCs have been initiated in the early 90s based mainly on the pioneering work of chaos synchronization in [35] using master-slave technique. Using this method, two chaotic systems (master and slave) with the same set of differential equations and parameters can be completely synchronized. The attractive features of chaotic signals, such as the high sensitivity to ICs and broadband spectrum, have led to large investments in CBSC for varied applications. In the following, we provide a brief review of the utilized chaotic modulation techniques for CBSC systems with coherent or noncoherent receivers. Multiuser chaotic communications are also considered for the multiple access channel. Then, the achieved physical security in CBSC systems is discussed.

\subsection{Chaotic Modulation Techniques}


In the literature, there are several modulation techniques used in chaotic communications which can be divided into coherent and noncoherent methods [21], [24].

Coherent Modulation Techniques. In coherent CBSC system, a synchronized copy of the chaotic signal, which is used by transmitter to modulate the users' data, is required at the receiver. Thus, the demodulation process is accomplished by comparing the received signal with the replica one that is available at the receiving end. The decision for which symbol was transmitted can be made according to the best level of achieved synchronization [21].

Chaotic Masking (CM). In this technique, the original message is added to the chaotic signal sequence. Then, the produced signal is transmitted through the communication channel. The original message can be restored then by subtracting the regenerated chaotic signal from the received signal. The security of this technique is considered poor since the process of adding a message to the chaotic signal will lead to a change in the power of the transmitted signal. Therefore, potential attackers may figure out the transmitted messages easily by detecting the power level changes of the transmitted signal [21], [25], [27].

Chaotic Modulation. The message in this case is injected directly into the chaotic system to alter its dynamics. The resultant chaotic signal will contain the analogue message which can be recovered using simple controllers at the receiver to detect the changes in the observed dynamic behavior of the chaotic signal. The prime disadvantage of this method is the need for new controller design for each chaotic system [21], [25]-[28].

Chaos Shift Keying (CSK). In this method, different chaotic systems are used for mapping each of the symbols, such that the size of the signal set is equal to the number of chaotic signal generators. At the receiver, the message symbol can be recovered according to the best synchronization achieved between the received signal and the regenerated chaotic signal. This method is considered as one of the finest schemes due to its robustness against noise effects and parameter mismatch [21], [27], [36], [37].

Chaos-Based Direct Sequence-CDMA. The chaotic based direct sequence code division multiple access (DS-CDMA) is also called CSK when a perfect synchronization is achieved. In this method, a chaotic sequence is used for the spreading of message symbols, where for every time interval a new chaotic code is generated. To extract the transmitted data, the synchronization unit is used to reconstruct the chaotic spreading sequence for the correlation process with the received signal. Finally, the correlated signal is compared with a threshold level in order to decide which bit was transmitted [21], [22], [32].

Noncoherent Modulation Techniques. There are many noncoherent CBSC systems in which there is no need for chaos synchronization. The demodulation process is performed by detecting the features of received chaotic signals.

Chaos On-Off Keying (COOK). This technique is known by its simplicity where the message is multiplied by the chaotic signal and transmitted to the receiver only for bit " 1 ". For bit " 0 ", the transmitter will be off and hence no emitted power [21], [25].

Chaotic Parameter Modulation (CPM). In this scheme, the message signal is used to modify a specific parameter in the chaotic drive system. Afterward, the drive signal will be sent to the 
receiver through the channel. At the receiver, the synchronization error between the received signal and the reconstructed chaotic states is used to recover the original message. This scheme is known to be robust against the noise effect with a high level of security [21], [27].

Differential Chaos Shift Keying (DCSK). In this technique, every bit's duration is divided into two equivalent slots. The first one is assigned for the reference chaotic signal while the second is used to send the reference signal or its inverted version depending on the bit being sent. The reference signal is sent through the same channel with the information signal, and hence no need to generate it at the receiver side. This method has attractive features like good resistance to linear/nonlinear channel distortions and no need for the channel state information (CSI). However, it suffers from serious problems such as low data rate, low-security level, variable bit energy, no support for mobility, and high energy consumption [21], [22], [29], [33].

\subsection{Multiuser Chaotic Communications}

The wideband property of chaotic signals is invested in multiple access (MA) techniques where the chaotic codes are used for data spreading [17], [22], [29], [31]-[33], [36]. In [31], a chaotic digital CDMA scheme was proposed by employing chaotic codes for data spreading instead of the pseudo-random codes. The achieved results have shown about two-fold channel capacity compared with conventional CDMA. Moreover, the works in [18] and [19] have considered the chaotic spreading codes in asynchronous DS-CDMA to enhance the system capacity. This comes due to the reduction of inter-user interference which resulted from the employment of the chaotic codes for data spreading. A coherent MA based on CSK with correlator receiver was proposed in [36]. The theoretical expression of bit-error-rate (BER) performance was derived and validated with the simulated results. Besides, it has been shown that as the number of users increases, the amount of induced interference among users will also increase and lead to deterioration in the BER performance. For instance, Figure 1 shows the BER performance of the CSK based MA scheme for chaotic code length $\beta=1000$. As can be seen, the decay in BER is clear as the number of users increases. In [33], another MA technique using DCSK was suggested and demonstrates similar BER performance for all users, while longer chaotic codes for data spreading have shown better error performance.

The investment of chaotic communications with multiple-input multiple-output (MIMO) techniques was also investigated in [19] and [23]. A secure chaotic MIMO (C-MIMO) communication system for messages with large amplitudes was introduced in [32]. It demonstrated larger capacity, higher quality, and improved physical layer security, but at the cost of increased decoding complexity.

\subsection{Security in Chaotic Communications}

The process of hiding messages by chaotic signals will result in an encrypted signal. At the receiver side, the decoding process is not possible without prior knowledge of the ICs, chaotic parameters, utilized code, and power fractions of the transmitted signal. Thus, potential intruders may not be able to demodulate the transmitted signals easily. As a result, chaotic communications provide physical layer security (PLS), where only the receiver with the right system parameters will be able to decode the signal. Therefore, chaotic modulations can take the place of the classical encryption and decryption techniques. In the latter scenarios, the encryption protocols can provide an upper-layer security (ULS) [6], [7], [13], [17]-[19]. 


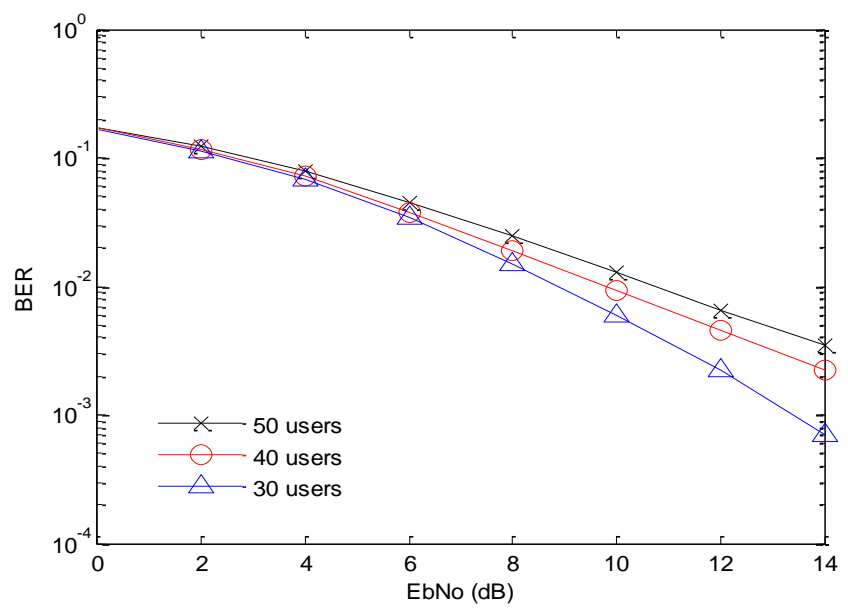

Fig. 1. Average BER of coherent CSK based MA over AWGN channel with $\beta=1000$ [36].

The ULS is mainly used in conventional communications, however, it might have serious problems under certain circumstances. Like in the case of 5G massive connectivity, the ULS protocols might become expensive, complicated, and not environment friendly due to high consumption power. The power consumption is due to the need for a two-way verification in addition to the signal processing burdens at the base station (BS) [6], [19], [38-40].

\section{NOMA Schemes}

In conventional OMA schemes, such as time division multiple access (TDMA), frequency division multiple access (FDMA), code division multiple access (CDMA), space division multiple access (SDMA), and orthogonal frequency division multiple access (OFDMA), the available resources are divided among users to mitigate the impact of co-channel interference. Though, these schemes fail to achieve the high capacity requirement for next-generation wireless networks. Therefore, NOMA is adopted in Long Term Evolution (LTE) standards for $4.5 \mathrm{G}$ and $5 \mathrm{G}$ systems to realize the critical needs of high connectivity and spectral efficiency [8], [10], [13], [14], [41], [42]. It can be realized through power-domain NOMA (PD-NOMA) and code-domain NOMA (CD-NOMA). These schemes are discussed in the following.

\subsection{PD-NOMA}

In PD-NOMA, the supported users are assigned different powers based on their channels, where users with poor channels' gains are assigned more power than users with strong channels. The near-far effect is invested in achieving higher spectral efficiency by making use of the natural variation of channel gains. Successive interference cancellation (SIC) can be used at the receiver to separate the signals of different users, where the strongest user will be detected first 
and its contribution is removed from the received superimposed signal, then other users will be detected successively according to their power levels [42].

\subsection{CD-NOMA}

In CD-NOMA, non-orthogonal spreading sequences are employed for the served users rather than the conventional orthogonal sequences in CDMA systems. For most CD-NOMA schemes, the message passing algorithm (MPA) can be used for multiuser detection (MUD) at the receiver. Currently, there are many CD-NOMA schemes like low-density spreading CDMA (LDS-CDMA), low-density spreading OFDM (LDS-OFDM), sparse-code MA (SCMA), and multiuser shared access (MUSA) [8], [10], [12], [16].

LDS-CDMA. The key concept of this scheme is to engage LDS sequences instead of the orthogonal sequences to mitigate the induced interference in each chip. Therefore, user overloading can be realized as the interference between multiple users is reduced [8], [10].

LDS-OFDM. In this technique, LDS-CDMA and OFDM are combined. The LDS sequences are used for spreading the data and the resulting chips are transmitted by employing a number of OFDM subcarriers [8], [10], [15].

Sparse Code Multiple Access (SCMA). The process of mapping and spreading are merged in this technique. Each user has a special codebook to map the data using the available sparse codewords. Then, the codewords of all users are multiplexed over shared orthogonal resources like OFDM subcarriers. SCMA is known to have a grant free transmission with the ability to support up to three times users more than the number of orthogonal resources. However, the codebook design is considered to be a tedious process [8], [10], [13], [16].

Multi-User Shared Access (MUSA). In this scheme, the transmitted data for a given user is multiplied by an identical, short, complex-valued, and low correlated spreading sequence. After spreading, the same orthogonal resources are used for the transmission of the users' data, and a codeword-level SIC can be used at the receiver side to separate the overlaid users' signals. MUSA is capable of achieving an outstanding system performance gain, especially for high values of user overloading factor [8], [12], [13].

\subsection{Other NOMA Schemes}

There are additional NOMA schemes like spatial division multiple access (SDMA), pattern division multiple access (PDMA), signature-based NOMA, interleaver-based NOMA, and spreading-based NOMA [10], [16].

Spatial Division Multiple Access (SDMA). This scheme uses the channel impulse responses (CIRs) of different users for distinguishing users instead of the user-specific spreading sequences. At the receiver, a precise CIR estimation is needed for a successful MUD which is a great challenge when the number of users is more than the receive antennas at BS [10], [15].

Pattern Division Multiple Access (PDMA). In PDMA, users' data are mapped to a group of resources based on the available patterns. Different resources can be used in this pattern like time, frequency, space, or mixture. The PDMA pattern can be represented in the form of a vector and its dimensions equal to the number of users and resources [13], [10], [16], [43]. 


\subsection{The Integration of NOMA and other Promising Schemes}

MIMO is one of the key techniques that invoked with NOMA to meet the high spectral efficiency requirements of 5G and beyond. In PD-NOMA, the power allocation depends on the channel conditions of each user which can be easily computed for single input scenarios. But, the channel gains of MIMO systems are represented in a matrix form which makes the comparison between channels and consequently the power allocation process very complex [44]. Therefore, beamforming technique is suggested as one of the efficient solutions to overcome this problem. At the BS, a number of transmitting antennas are used to form a number of beams, and PD-NOMA can be used within each beam. At the receiver, spatial filtering can be used for cancelling the inter-beam interference followed by SIC to eliminate the inter-user interference [2], [5], [9], [45]-[47].

In PD-NOMA with large number of users, the decoding complexity becomes considerably high due to SIC process that causes also possible delay and error propagation. Therefore, multicarrier NOMA technique is adopted as one of the efficient solutions to realize a balance between system complexity and desired performance. In such a technique, users are divided into groups, where each group of users will be served by the same block of orthogonal resources based on the NOMA principle. Besides, different blocks of the orthogonal resources are assigned to different groups. Within each group, the intra-group interference is avoided based on NOMA principle while the inter-group interference is mitigated by employing a number of subcarriers like the case in OFDMA. Consequently, this procedure can lead to an enhancement in system capacity at low complexity since the number of users served by the same orthogonal resource is reduced [1], [20].

NOMA is integrated also with chaotic communications as in [17] where a sparse chaotic code multiple access (SCCMA) is achieved. In this scheme, the chaotic signals are employed for the construction of codebooks to increase the number of supported users without the need for complex codebooks pre-design (as in the conventional SCMA). In [7] and [18], a downlink and uplink schemes for chaos NOMA (C-NOMA) schemes were presented. C-MIMO is merged also with NOMA to provide a system having higher capacity and improved security. In these systems, the user's data is modulated by chaotic signals then superimposed in the power domain using a specific scheduling algorithm. An efficient joint maximum-Likelihood sequence estimation (MLSE) is used for signal demodulation at the cost of high complexity that as the number of users increases. Figure 2 demonstrates the average BER performance of C-NOMA compared with BPSK-NOMA and chaos-OFDMA (C-OFDMA). It was shown that C-NOMA achieves better performance than the unencrypted BPSK-NOMA (about $5 \mathrm{~dB}$ gain at BER of $10^{-5}$ ) due to the effect of coding gain provided by C-MIMO.

\section{Wireless Nonorthogonal Chaotic Communication (WNCC)}

The hybrid combination of chaotic modulation/spreading techniques and NOMA configurations is denoted in this survey as WNCC schemes. To clarify the basic working principles of WNCC schemes, a simple scenario of 2 users communicating simultaneously with a common BS over uplink wireless fading channel is shown in Figure 3.

The user's data $b_{i} ; i=1,2$ from digital information source is modulated using chaotic code sequence $\mathbf{c}$ of length $\beta$. The employed chaotic codes by the users can be generated simply from chaotic drive systems such as Lorenz and Chua circuits [25], [26], [30]. 


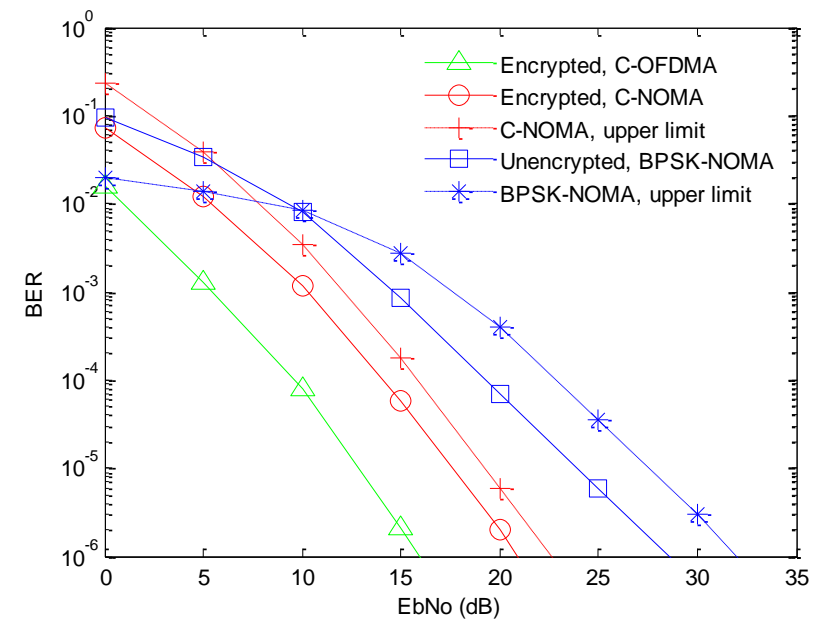

Fig. 2. Average BER performance of C-NOMA for a receive antenna at the cell edge [7].

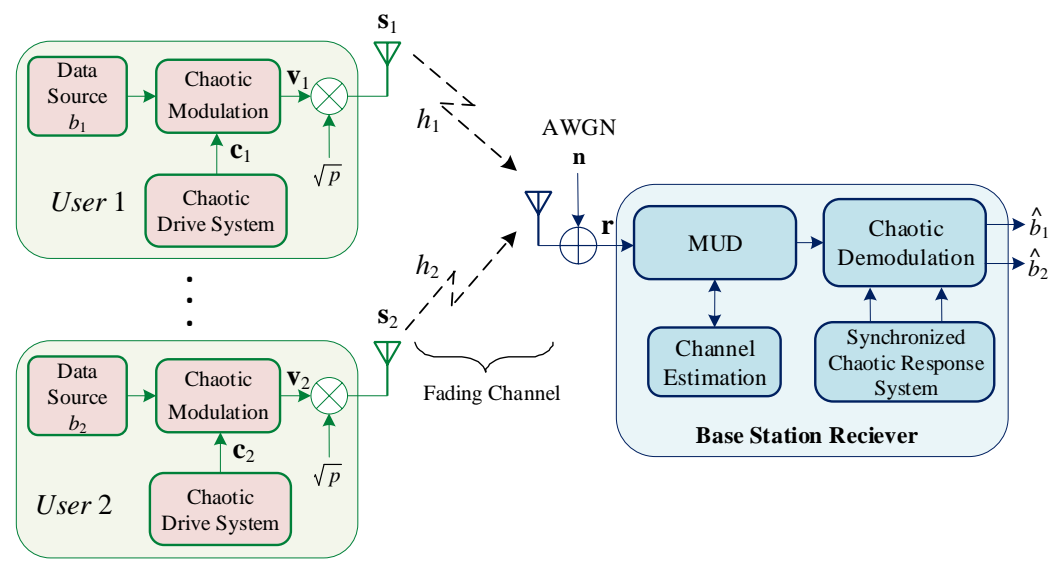

Fig. 3. System model for a simple scenario of uplink WNCC with 2 users communicating simultaneously with a common base station over wireless fading channel.

The modulated signal vector $\mathbf{v}_{i}$ is transmitted as $\mathbf{S}_{i}$ with an average power $p$ over large-scale (path-loss) and small-scale Rayleigh fading (time-varying attenuation) channel $h_{i}$ and additive white Gaussian noise (AWGN) vector $\mathbf{n}$ to the BS receiver. The received signal vector $\left(\mathbf{r}=h_{1} \mathbf{s}_{1}+h_{2} \mathbf{s}_{2}+\mathbf{n}\right)$ is used for MUD to estimate the user's data as $\hat{b}_{i}$.

The presented WNCC system can attain the desired PLS owing to the adoption of chaotic modulation. Furthermore, the MA can be realized as code-domain WNCC (CD-WNCC) for equal power allocation, power-domain WNCC (PD-WNCC) for distinct power allocation, and 
power-code-domain WNCC (PCD-WNCC). Figure 4 illustrates a clear vision of the different realizations of WNCC schemes with $K$ users more than the available orthogonal resources $N$.

In scenario 1 (S1) which represents a possible CD-WNCC system, equal power allocation $\left(p_{1}=p_{2}=\cdots=p_{Q}=p\right)$ is used with different chaotic codes $\left(\mathbf{c}_{1} \neq \mathbf{c}_{2} \neq \cdots \neq \mathbf{c}_{Q}\right)$ for users $\left(u_{1}, u_{2}, \cdots, u_{Q}\right)$, respectively to achieve the required signal separation for the multiple access channel. The ML or matched filter (MF) can be used as a MUD at the receiver. We have implemented this scenario in our recent work [30] and an example of the achieved BER results is demonstrated in Figure 5. From the results, it can be seen that CD-WNCC is able to achieve both of the multiple access reliability and PLS compared with the reference systems.

On the other hand, Scenario 2 (S2) represents the PD-WNCC system which employs the same chaotic code for all the users $\left(\mathbf{c}_{1}=\mathbf{c}_{2}=\cdots=\mathbf{c}_{Q}=\mathbf{c}\right)$ with different power levels for supported users as $p_{1}>p_{2}>\cdots>p_{Q}$. Due to the existence of power differences among multiple users, the SIC technique can be used at the receiver for signal detection.

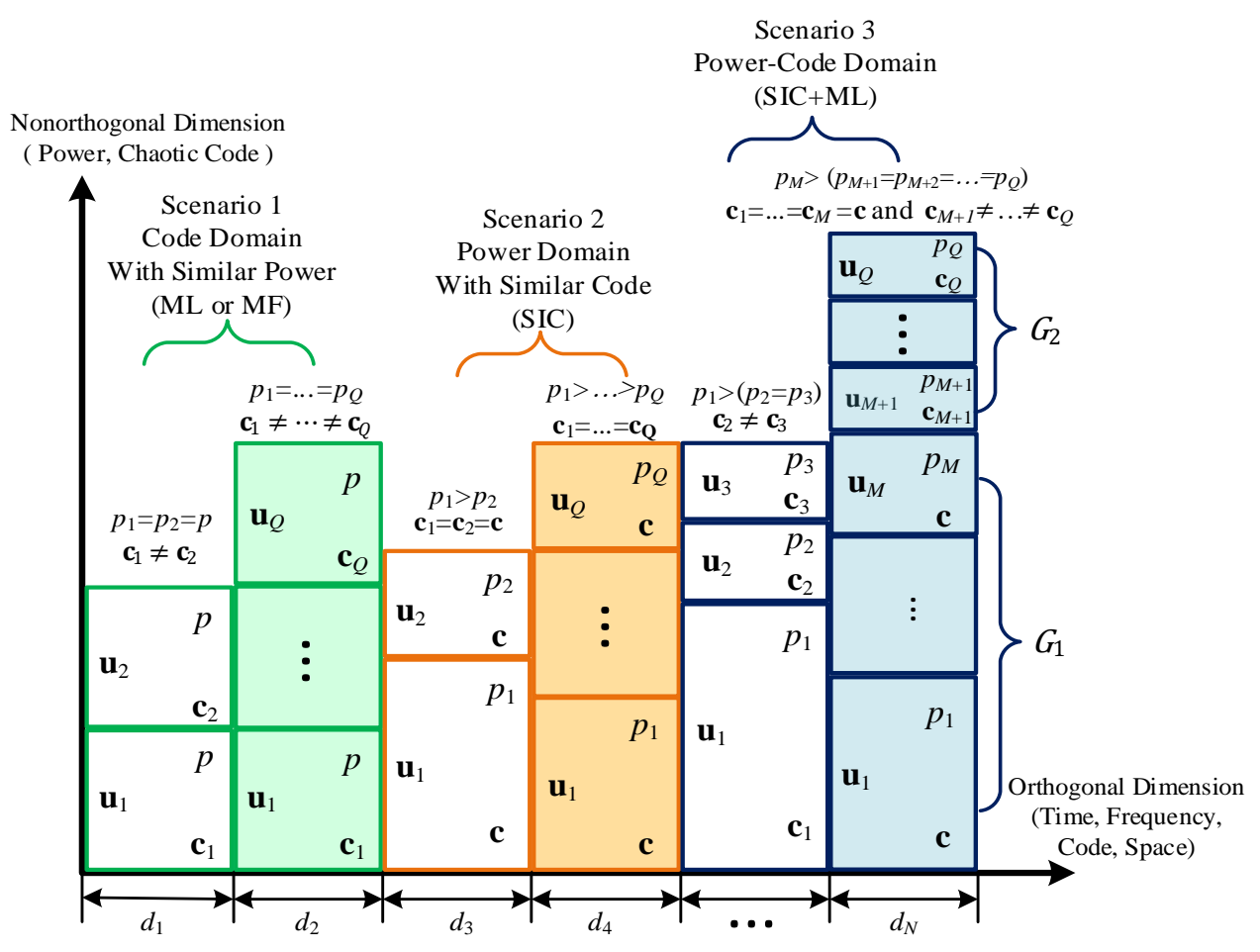

Fig. 4. A vision on the possible of WNCC schemes with $K$ supported users more than the available orthogonal resources $N$

In the third scenario (S3), PCD-WNCC can be realized by utilizing different power levels and different codes. For example, the served users can be divided into two groups $G_{1}$ and $G_{2}$ as demonstrated in Figure 4. For users within $G_{1}$, different power assignments are used $\left(p_{1}>p_{2}>\cdots>p_{M}\right)$ with the same code as $\mathbf{c}_{1}=\mathbf{c}_{2}=\cdots=\mathbf{c}_{M}$. In the meantime, users in $G_{2}$ 
will be assigned a similar power level $\left(p_{M+1}=p_{M+2}=\cdots=p_{Q}\right)$ and distinct codes as $\mathbf{c}_{M+1} \neq$ $\mathbf{c}_{M+2} \neq \cdots \neq \mathbf{c}_{Q}$. Note that the minimum assigned power for users in $G_{1}$ should be greater than the allocated power for users within $G_{2}$ such that $p_{M}>\left(p_{M+1}=p_{M+2}=\cdots=p_{Q}\right)$. According to these power constraints, the decoding process at the receiver can take place on two stages. In the first stage, SIC is applied to distinguish $G_{1}$ from $G_{2}$ due to the presence of power difference between these two groups. The second stage of MUD can be performed by applying SIC for $G_{1}$ users and ML for $G_{2}$ users.

Table 1 summarize the discussed scenarios of WNCC schemes with their power/code constraints and MUD techniques. The formerly mentioned realizations can be considered potential candidates for $5 \mathrm{G}$ communications since they harness all NOMA traits while holding PLS requirement. It should be noted that the implementation of classical ULS techniques are costly in massive connectivity due to ultra-high complexity and increased power consumption.

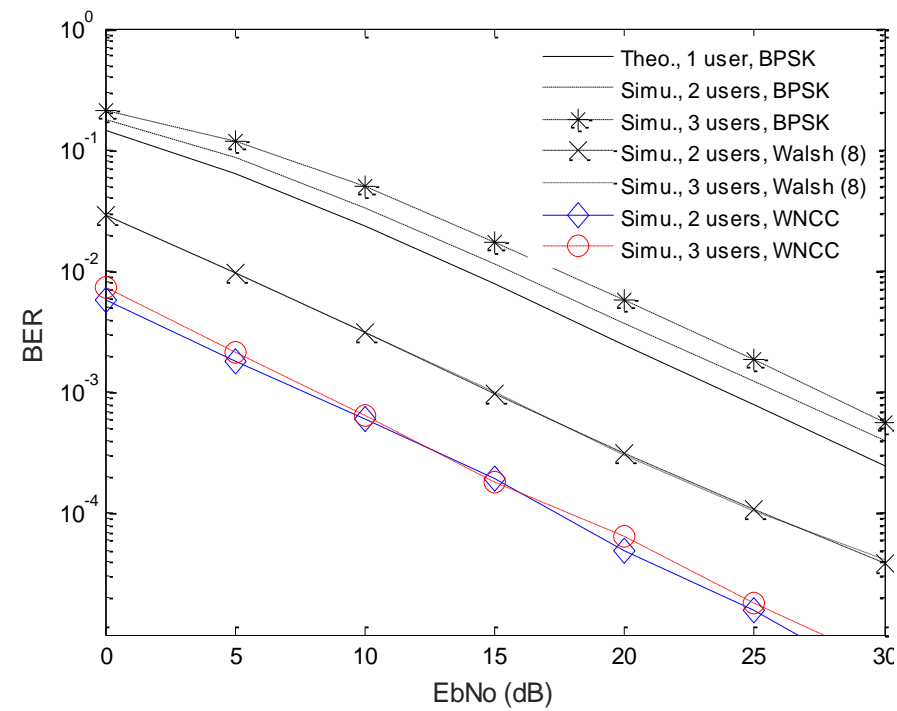

Fig. 5. Average BER performance of CD-WNCC system of two and three users over Rayleigh fading channel when $\beta=50$.

Table 1. Summary of the possible realizations of WNCC with maximum of $K=N Q$ users.

\begin{tabular}{c|c|c|c}
\hline Scenario & Power-Domain Conditions & Code-Domain Conditions & $\begin{array}{c}\text { MUD } \\
\text { Method }\end{array}$ \\
\hline S1 & $p_{1}=p_{2}=\cdots=p_{Q}=p$ & $\mathbf{c}_{1} \neq \mathbf{c}_{2} \neq \cdots \neq \mathbf{c}_{Q}$ & $\begin{array}{c}\text { ML or } \\
\text { MF }\end{array}$ \\
\hline S2 & $p_{1}>p_{2}>\cdots>p_{Q}$ & $\mathbf{c}_{1}=\mathbf{c}_{2}=\cdots=\mathbf{c}_{Q}=\mathbf{c}$ & SIC \\
\hline S3 & $\begin{array}{c}p_{1}>p_{2}>\cdots>p_{M} \\
p_{M}>\left(p_{M+1}=p_{M+2}=\cdots=p_{Q}\right)\end{array}$ & $\begin{array}{c}\mathbf{c}_{1}=\mathbf{c}_{2}=\cdots=\mathbf{c}_{M} \\
\mathbf{c}_{M+1} \neq \mathbf{c}_{M+2} \neq \cdots \neq \mathbf{c}_{Q}\end{array}$ & $\begin{array}{c}\text { SIC } \\
+ \\
\text { ML }\end{array}$ \\
\hline
\end{tabular}




\section{Challenges and Practical Considerations for WNCC}

Since the implementation of WNCC is based on the integration of CBSC and NOMA, the practical concerns of each of these two schemes will be reflected to the overall system design, in addition to other new considerations. The most important issues are highlighted as follows:

- Maintaining chaos synchronization is one of the critical issues in WNCC systems that employ coherent MUD technique. In general, there are two approaches to achieve the required synchronization. The first one requires regeneration of the same chaotic signal used at the transmitter with the same ICs. This method is not preferable since any small change in ICs will lead to producing a different chaotic signal, and then a high possibility of link failure. For the second method, the chaotic signal is generated and stored in both the transmitter and receiver. So, the problem of high sensitivity to IC can be mitigated at the cost of feedback and additional memory requirements [21], [25], [35]. Many updates and improvements in the field of chaos synchronization such as the methods in [27] can be extended also for practical WNCCs.

- Energy-efficient chaotic circuit designs are required to attain the constraints for green communications [2], [38]-[40] with desired information security [6]. The available circuits for chaos generation in many well-known publications like [21], [27], and [35] can be further improved to achieve this important target.

- Error propagation is another difficulty when SIC receiver is used for MUD in the WNCC system. It is obvious that once a user is decoded erroneously, this error will propagate to the following users which has a direct impact to degrade the error performance. In this context, using chaotic codes with large lengths can help in compensating the effect of error propagation at the cost of additional complexity. Therefore, a proper design that considers the trade-off between these factors should be investigated [7], [30].

- When other OMA schemes are integrated with WNCC, efficient and low complexity user grouping and power control techniques are needed to satisfy the target quality of service (QoS) and other important issues such as maximum channel capacity and fairness. This requires an accurate estimation of CSI which is necessary for MUD at the same time [5], [10], [15], [48]. Signaling overhead should be considered due to its direct relation to degrading the data rate. Low complexity MUDs are also considered to reduce the processing time (i.e. delay) and consumed power for green communications [1], [2], [38], [39].

\section{Future Research Directions of WNCC}

From the presented details and discussions in this paper, several potential and important research directions can be extracted to extend the state-of-the-art of WNCC for future applications. It includes, but not limited to, the following points:

- The extension of WNCC with other OMA such as MIMO and OFDMA schemes is an interesting topic to enhance user connectivity and spectral efficiency. This requires intensive studies for the optimal/suboptimal resource allocation and user grouping strategies to satisfy QoS, network targets, and user fairness [1], [9], [13], [19].

- The trade-offs between system performances, MUD complexity, overhead requirements, and desired security level are a very important topic to be investigated for WNCCs with OMA schemes [1], [2], [49], [50]. 
- The impact of channel estimation error and hardware impairments on the performance and robustness of WNCC systems need to be carefully investigated.

- Closed-form analytical expressions of the error rate and capacity of WNCC are very important and needed to highlight the upper and lower benchmark limits for the actual system performance [9], [49].

- Prototype designs and field tests are also required to assess the performance and capabilities of WNCC systems in real-time applications towards the potential adoption in 5G communication networks.

\section{Conclusion}

In this paper, concentrated technical background reviews on the existing CBSC and NOMA schemes have been presented with their main features, results and applications in wireless communications. The integration of these promising techniques in WNCC designs is investigated highlighting the possible opportunities based on code and/or power domains. The sample results of CD-WNCC demonstrated the effectiveness of the proposed design in terms of user connectivity, BER performance, and physical layer security. Major implementation challenges and future research directions for WNCC systems are highlighted. It is expected that PCD-WNCC will provide excellent performance and superior physical layer security compared with the other investigated configurations. This may lead to the possible adoption of WNCCs in $5 \mathrm{G}$ networks and beyond.

\section{References}

[1] Al-Hussaibi W., Ali F.: Efficient user clustering, receive antenna selection, and power allocation algorithms for massive MIMO-NOMA systems. IEEE Access. Vol. 7, No. 6, pp. 31865-31882 (2019) [2] Al-Hussaibi W., Ali F.: Performance-complexity tradeoffs of MIMO-NOMA receivers towards green wireless networks. Proc. 30th IEEE PIMRC'2019. pp. 654-659, Istanbul, Turkey (2019)

[3] Lien S.-Y., Shieh S.-L., Huang Y., Su B., Hsu Y.-L., Wei H.-Y.: 5G new radio: waveform, frame structure, multiple access, and initial access. IEEE Commun. Mag. Vol. 55, No. 6, pp. 64-71 (2017)

[4] Parkvall S., Dahlman E., Furuskar A., Frenne M.: NR: the new 5G radio access technology. IEEE Commun. Standards Mag. Vol. 1, No. 4, pp. 24-30 (2017)

[5] Al-Hussaibi W., Ali F.: Group layer MU-MIMO for 5G wireless systems. Telecommunication Systems. Vol. 70, No. 4, pp. 525-540 (2019)

[6] Hamamreh J., Furqan H., Arslan H.: Classifications and applications of physical layer security techniques for confidentiality: A comprehensive survey. IEEE Commun. Surveys \& Tutorials. Vol. 21, No. 2, pp. 1773-1828 (2019)

[7] Horiike N., Okamoto E., Yamamoto T.: A downlink non-orthogonal multiple access scheme having physical layer security. EURASIP Journal on Wireless Communications and Networking. Vol. 2018, No. 205, pp. 1-13 (2018)

[8] Dai L., Wang B., Yuan Y., Han S., I C-L, Wang Z.: Non-orthogonal multiple access for 5G: solutions, challenges, opportunities, and future research trends. IEEE Commun. Mag., Vol. 53, No. 9, pp. 74-81 (2015)

[9] Al-Hussaibi W.: Optimal cluster formation and power control for high connectivity wireless MIMONOMA applications. Electronics Letters. Vol. 55, No. 20, pp. 1110-1112 (2019) 
[10] Dai L., Wang B., Ding Z., Wang Z., Chen S., Hanzo L.: A survey of non-orthogonal multiple access for 5G. IEEE Commun. Surveys and Tutorials. Vol. 20, No. 3, pp. 2294-2323 (2018)

[11] Sari H., Maatouk A., Caliskan E., Assaad M., Koca M., Gui G.: On the foundation of NOMA and its Application to 5G cellular networks. Proc. IEEE Wireless Communications and Networking Conference (WCNC). pp. 1-6, Barcelona, Spain (2018)

[12] Ding Z., Liu Y., Choi J., Sun Q., Elkashlan M., I C.-L., Poor H.V.: Application of non-orthogonal multiple access in LTE and 5G Networks. IEEE Commun. Mag. Vol. 55, No. 2, pp. 185-191 (2017)

[13] Ding Z., Lei X., Karagiannidis G., Schober R., Yuan J., Bhargava V.: A survey on non-orthogonal multiple access for $5 \mathrm{G}$ networks: research challenges and future trends. IEEE J. Sel. Areas Commun. Vol. 35, No. 10, pp. 2181-2195 (2017)

[14] Chen X., Zhang Z., Zhong C., Jia R., Ng D.: Fully non-orthogonal communication for massive access. IEEE Trans. Commun. Vol. 66, No. 4, pp. 1717-1731 (2018)

[15] Islam S., Avazov N., Dobre O., Kwak K.-S.: Power-domain non-orthogonal multiple access (NOMA) in 5G systems: potentials and challenges. IEEE Commun. Surveys \& Tutorials. Vol. 19, No. 2, pp. 721-742 (2017)

[16] Wu Z., Lu K., Jiang C., Shao X.: Comprehensive study and comparison on 5G NOMA schemes. IEEE Access. Vol. 6, pp. 18511-18519 (2018)

[17] Okamoto E., Horiike N., Yamamoto T.: Sparse chaos code multiple access scheme achieving larger capacity and physical layer security. Proc. 20th International Symposium on Wireless Personal Multimedia Communications (WPMC). pp. 604-610, Bali, Indonesia (2017)

[18] Masuda Y., Okamoto E., Ito K., Yamamoto T.: An uplink non-orthogonal multiple access scheme having physical layer security based on chaos modulation. Proc. International Conference on Information Networking (ICOIN). pp. 136-140, Kuala Lumpur, Malaysia (2019)

[19] Okamoto E.: A chaos MIMO transmission scheme for secure communications on physical layer. Proc. IEEE 73rd Vehicular Technology Conference (VTC Spring). pp. 1-5, Yokohama, Japan (2011)

[20] Chen X., Zhang Z., Zhong C., Ng D., Jia R.: Exploiting inter-user interference for secure massive non-orthogonal multiple access. IEEE J. Sel. Areas Commun. Vol. 36, No. 4, pp. 788-801 (2018)

[21] Kaddoum G.: Wireless chaos-based communication systems: a comprehensive survey. IEEE Access. Vol. 4, pp. 2621-2648 (2016).

[22] Tam W., Lau F., Tse C.: A multiple access scheme for chaos-based digital communication systems utilizing transmitted reference. IEEE Trans. Circuits and Systems I: Regular Papers. Vol. 51, No. 9, pp.1868-1878 (2004)

[23] Zheng G., Boutat D., Floquet T., Barbot J.: Secure communication based on multi-input multioutput chaotic system with large message amplitude. Chaos, Solitons and Fractals. Vol. 41, No. 3, pp. 1510-1517 (2009)

[24] Ren H.-P., Baptista M., Grebogi C.: Wireless communication with chaos. Physical Review Letters Vol. 110, No. 18, pp. 1-5 (2013)

[25] Al-Hussaibi W.: Filtering effects on the synchronization and error performance of promising wireless chaos-based secure communications. Wireless Networks. Vol. 21, No. 6, pp. 1957-1967 (2015)

[26] Jovic B.: Chaos-based BPSK communication system. Electronics Letters. Vol. 51, No. 8, pp. 630$632(2015)$

[27] Al-Hussaibi W.: Effect of filtering on the synchronization and performance of chaos-based secure communication over Rayleigh fading channel. Commun. in Nonlinear Science and Numerical Simulation. Vol. 26, No. 1-3, pp. 87-97 (2015)

[28] Al-Hussaibi W., Alsmael J., Türkmen M.: On the chaos synchronization in CBSC systems over realistic wireless channels. Proc. 16th SSD'19-CSP. pp. 654-659, Istanbul, Turkey (2019)

[29] Tam W., Lau F., Tse C.: Analysis of bit error rates for multiple access CSK and DCSK communication systems. IEEE Trans. Circuits and Systems I: Fundamental Theory and Applications. Vol. 50, No. 5, pp. 702-707 (2003)

[30] Almusawi I., Al-Hussaibi W., Tahir Y.: Chaos-based NOMA for secure wireless communications over Rayleigh fading channels. Accepted in IMDC-SDSP 2020, Turkey (2020) 
[31] Yang T., Chua L.: Chaotic digital code-division multiple access (CDMA) communication systems. International Journal of Bifurcation and Chaos. Vol. 7, No. 12, pp. $2789-2805$ (1997)

[32] Mazzini G., Setti G., Rovatti R.: Chaotic complex spreading sequences for asynchronous DSCDMA. Part I: system modeling and results. IEEE Trans. Circuits Systems I: Fundamental Theory and Applications. Vol. 44, No. 10, pp. 937-947 (1997)

[33] Lau F., Yip M., Tse C., Hau S.: A multiple-access technique for differential chaos-shift keying. IEEE Trans. Circuits and Systems I: Fundamental Theory and Applications. Vol. 49, No. 1, pp. 96-104 (2002)

[34] Harb A., Al-Hussaibi W., Khadra L.: Effects of filtering chaotic signals of power electronic circuit. International Journal of Modeling and Simulation. Vol. 23, No. 2, pp. 129-134 (2003)

[35] Pecora L., Carroll T.: Synchronization in chaotic systems. Physical Review Letters. Vol. 64, No. 8, pp. 821-824 (1990)

[36] Tam W., Lau F., Tse C., Yip M.: An approach to calculating the bit-error rate of a coherent chaosshift-keying digital communication system under a noisy multiuser environment. IEEE Trans. Circuits and Systems I: Fundamental Theory and Applications. Vol. 49, No. 2, pp. 210-223 (2002)

[37] Soujeri E., Kaddoum G., Herceg M.: Design of an initial condition-index chaos shift keying modulation. Electronics Letters. Vol. 54, No. 7, pp. 447-449 (2018)

[38] Chih-Lin I., Rowell C., Han S., Xu Z., Li G., Pan Z.: Toward green and soft: A 5G perspective. IEEE Commun. Mag. Vol. 52, No. 2, pp. 66-73 (2014)

[39] Gandotra P., Jha R., Jain S.: Green communication in next generation cellular networks: A survey. IEEE Access. Vol. 5, pp. 11727-11758 (2017)

[40] Lei L., Yuan D., Varbrand P.: On power minimization for non-orthogonal multiple access (NOMA). IEEE Commun. Letters. Vol. 20, No. 12, pp. 2458-2461 (2016)

[41] Ding Z., Yang Z., Fan P., Poor H.: On the performance of non-orthogonal multiple access in 5G systems with randomly deployed users. IEEE Signal Process. Letters. Vol. 21, No. 12, pp. 1501-1505 (2014)

[42] Zhang N., Wang J., Kang G., Liu Y.: Uplink nonorthogonal multiple access in 5G systems. IEEE Commun. Letters. Vol. 20, No. 3, pp. 458-461 (2016)

[43] Chen S., Ren B., Gao Q., Kang S., Sun S., Niu K.: Pattern division multiple access-a novel nonorthogonal multiple access for fifth-generation radio networks. IEEE Trans. Vehicular Technology. Vol. 66, No. 4, pp. 3185-3196 (2017)

[44] Ding Z., Adachi F., Poor H.: The Application of MIMO to non-orthogonal multiple access. IEEE Trans. Wireless Commun. Vol. 15, No. 1, pp. 537-552 (2015)

[45] Chi Y., Liu L., Song G., Yuen C., Guan Y., Li Y.: Practical MIMO-NOMA: low complexity and capacity-approaching solution. IEEE Trans. Wireless Commun. Vol. 17, No. 9, pp. 6251-6264 (2018) [46] Al-Hussaibi W., Ali F.: Constellation constrained multiuser multiple-input multiple-output for high capacity and error performance over correlated fading channels. Wireless Communications and Mobile Computing. Vol. 16, No. 6, pp. 717-732 (2016)

[47] Al-Hussaibi W., Ali F.: Extending the user capacity of MU-MIMO systems with low detection complexity and receive diversity. Wireless Networks. Vol. 24, No. 6, pp. 2237-2249 (2017)

[48] Maatouk A., Caliskan E., Koca M., Assaad M., Gui G., Sari H.: Frequency-domain NOMA with two sets of orthogonal signal waveforms. IEEE Commun. Letters. Vol. 22, No. 5, pp. 906-909 (2018) [49] Al-Hussaibi W., Ali F.: A closed-form approximation of correlated multiuser MIMO ergodic capacity with antenna selection and imperfect channel estimation. IEEE Trans. Vehicular Technology. Vol. 67, No. 6, pp. 5515-5519 (2018)

[50] Timotheou S., Krikidis I.: Fairness for non-orthogonal multiple access in 5G systems. IEEE Signal Processing Letters. Vol. 22, No. 10, pp. 1647-1651 (2015) 